\title{
Can Different Complex Training Improve the Individual Phenomenon of Post-Activation Potentiation?
}

\author{
by \\ Zong-Rong Chen ${ }^{1}$, Shin-Liang Lo ${ }^{2}$, Min-Hsien Wang ${ }^{2}$, Ching-Fang Y ${ }^{2}$, \\ Hsien-Te Peng ${ }^{2}$
}

\begin{abstract}
The aims of the present study were (a) to determine whether the two types of complex training and vibration complex training would improve the individual phenomenon of post-activation potentiation (PAP) for every athlete in a team setting; and (b) to compare the acute effect of resistance and plyometric exercise, whole body vibration, complex training and vibration complex training on vertical jump performance. The participants were ten male division I college volleyball and basketball players. They were asked to perform three vertical jumps as a pre-test and were then randomly assigned to one of five PAP protocols, resistance exercise using half squat exercise, plyometric exercise using drop jumps with individualized drop height, whole body vibration using squats on a vibration plate, complex training combining resistance exercise with plyometric exercise, vibration complex training combining whole body vibration with plyometric exercise. Three vertical jumps were performed four minutes after the PAP protocol as a post-test. A two-way repeated-measures analysis of variance was used to examine the differences among the five PAP protocols and between the two testing times. Our results showed that the post-test results were significantly improved compared to the pre-test for the vertical jump height $(p=.015)$ in all PAP protocols. There was, however, an individual phenomenon of PAP in the response to all PAP protocols. In conclusion, this study found that resistance and plyometric exercise, whole body vibration, complex training and vibration complex training induce similar group PAP benefits. However, some athletes decreased their performances in some of the exercises in the study. Therefore, it is not recommended for coaches to arrange the exercises in a team setting.
\end{abstract}

Key words: plyometric exercise, resistance exercise, whole body vibration, warm-up.

\section{Introduction}

Vertical jump (VJ) ability has been shown to be an important factor in the success or failure of sports performances (e.g. high, long and triple jumps) (Chattong et al., 2010). Researchers, coaches and athletes often utilise the VJ to examine muscle performance of the lower extremity and pursue improved VJ performances via exercise and/or training in sports. Previous studies have demonstrated that chronic heavy load exercise (e.g. 1 to 5 repetitions max [RM] squat, drop jump and whole body vibration) with appropriate prescription can improve VJ performance (Arabatzi et al., 2010; Byrne et al.,
2010; Wang et al., 2014). For acute improvement, some researchers and coaches have used heavy load exercise as a warm-up protocol to immediately enhance the following VJ performance (Chen et al., 2013; Cormie et al., 2006; Kilduff et al., 2007; Lamont et al., 2010; Gołaś et al., 2016).

An improved performance following heavy load exercise is known as the phenomenon of post-activation potentiation (PAP) (Kilduff et al., 2007). There are two proposed physiological mechanisms for PAP: 1) regulation of myosin light chain phosphorylation can increase the

1 - Graduate Institute of Sport Coaching Science Chinese Culture University, Taipei, Taiwan.

2 - Department of Physical Education Chinese Culture University, Taipei, Taiwan. 
sensitivity of actin-myosin for calcium ions $(\mathrm{Ca} 2+)$, alter myosin head structure and produce higher cross-bridge force output; and 2) muscle pre-contraction can increase excitation potential across the spinal cord resulting in increased motor unit recruitment (Kilduff et al., 2007). The excitation can increase postsynaptic potentials by lasting for several minutes and subsequently enhance force generation. Nevertheless, previous studies have demonstrated that heavy load exercise does not only produce PAP, but also induces muscular fatigue (Kilduff et al., 2007). Therefore, muscle performance following heavy load exercise depends on the balance between PAP and fatigue (Kilduff et al., 2007).

Previous studies have attempted to use different conditioning exercises (e.g. resistance exercise [RE], plyometric exercise [PE] and whole body vibration [WBV]) to increase the PAP leading motor performance (e.g. VJ performance); however, the results obtained are contradictory. Some studies have shown a subsequent improvement in VJ following RE, PE and WBV (Chen et al., 2013; Cormie et al., 2006; Lamont et al., 2010; McCann and Flanagan, 2010). In contrast, other studies have found no subsequent improvement in VJ following RE, PE and WBV (Dallas et al., 2014; Jensen and Ebben, 2003; SaezSaez de Villarreal et al., 2007). The discrepancies between these studies have been, at least in part, explained by the different type of conditioning exercise and/or different volumes and intensities of the conditioning exercises with a different recovery time (Miarka et al., 2011; SaezSaez de Villarreal et al., 2007; Till and Cooke, 2009; Gołaś et al., 2016).

Achieving peak VJ performance requires multifaceted components such as maximal and explosive strength through different conditioning exercise development (Arabatzi et al., 2010; McLellan et al., 2011). Therefore, single conditioning exercises (RE and PE) may not be as effective as complex training (combined RE with PE using biomechanically similar exercise, CT) for PAP leading to improvement of VJ performance. For example, RE includes high force and low movement velocity which may create conditions appropriate for PAP leading to maximal strength; $\mathrm{PE}$ includes low force and high movement velocity which may create conditions appropriate for PAP leading to explosive strength (Hanson et al., 2007; McCann and Flanagan, 2010; Tillin and Bishop, 2009). Theoretically, using CT during the same session can improve both maximal and explosive strength by combining the specificity of RE and PE. Previous studies have also confirmed that chronic CT produced significantly greater improvement in VJ performance than chronic single conditioning exercise (RE and PE) (Fatouros et al., 2000; Kotzamanidis et al., 2005). However, to date, it is unknown whether CT would induce a greater PAP effect on VJ performance as compared to single conditioning exercise (RE and PE). Moreover, previous studies demonstrated that WBV increased intra-muscle temperature (Cochrane et al., 2008) and not only induced PAP benefit, but also improved resistance to fatigue (Barroso et al., 2013). Thus, in the present study, we combined WBV with PE as vibration complex training (VCT) and expected to increase resistance to fatigue during PE following WBV to induce a greater PAP effect.

Previous studies showed that PAP was an individual phenomenon (Comyns et al., 2006; Lim and Kong, 2013; McCann and Flanagan, 2010; Weber et al., 2008) which was suggested to be induced according to participants' physical characteristics (Robbins, 2005). These researchers found that participants responded differently to protocols depending on the type of conditioning exercise, recovery time, volume and intensity. Some participants benefited from PAP, whereas others worsened their performances (Lim and Kong, 2013; McCann and Flanagan, 2010; Weber et al., 2008). Therefore, using a uniform protocol might be contraindicated to induce PAP in a team setting. In order to resolve the problem, previous studies suggested that the use of PAP protocols to improve performance should examine the effectiveness of different PAP protocols based on individual predispositions (Lim and Kong, 2013; McCann and Flanagan, 2010; Weber et al., 2008). However, it would be time consuming and challenging for coaches particularly when working in a team setting. To the best of our knowledge, there was no uniform protocol of those conditioning exercises that could improve performance of every athlete in a team setting. Moreover, previous researchers examined PAP effects using only a single type of conditioning exercise which showed the inconsistent outcomes of PAP (Comyns et al., 2006; Lim and Kong, 2013; 
McCann and Flanagan, 2010; Weber et al., 2008).

Based on these aforementioned reasons, it was legitimate to speculate that CT and VCT inducing PAP leading to enhanced VJ performance would be better than a single conditioning exercise and improve the individual phenomenon of PAP. Thus, the aims of the present study were (a) to determine whether CT and VCT would improve the individual phenomenon of PAP of every athlete in a team setting; and (b) to compare the acute effect of single conditioning exercise (RE, PE and WBV), CT and VCT on vertical jump performance.

\section{Material and Methods}

Design

A repeated-measures design was used to compare the acute effects of complex training $(\mathrm{CT})$, vibration complex training (VCT) and single conditioning exercise (PE, WBV and RE) on VJ performance. This study combined the aforementioned RE and PE for CT and combined WBV and PE for VCT. VJ height was measured four minutes after completion of the exercise as dependent variables to assess PAP effect (Comyns et al., 2006).

\section{Participants}

Ten male division I college volleyball and basketball players (age: $21.1 \pm 2.1$ years; body height: $181.8 \pm 7.2 \mathrm{~cm}$; body mass: $77.2 \pm 13.7 \mathrm{~kg}$; training experience: $8.0 \pm 3.2$ years) agreed to participate in this study. All participants were familiar with RE, PE and WBV, and were free from any musculoskeletal injury and cardiovascular - respiratory diseases. They were able to lift at least one and a half times their body weight during the squat exercise and were tested in the competition phase of their annual training cycle (training sessions five times a week). Written informed consent was acquired before participation. The study was approved by the Ethical Committee of the Chinese Culture university.

\section{Procedures}

The participants were asked to refrain from strenuous physical activity, alcohol, caffeine, additional supplementation and food intake two hours prior to all testing sessions. In addition, they were also asked to maintain their daily regular habits. It took one day to familiarise the athletes with the experimental protocol and to determine their 5 RM squat loads for RE and individualized drop heights for the PE drop jump before testing. The standardised footwear (Model s.y.m. B9025, LurngFurng, Inc., Taipei, Taiwan) was used to control for different shoe-sole absorption properties. The experiment was conducted in a sport biomechanical laboratory. The same examiner collected all data.

Each participant's $5 \mathrm{RM}$ squat was determined by the guidelines of the National Strength and Conditioning Association (Baechle and Earle, 2008). Briefly, 5 RM was defined as the maximal load that the participant could successfully complete for five repetitions with correct technique. To examine the $5 \mathrm{RM}$ squat load, the participants attempted five repetitions (each repetition to $90^{\circ}$ of knee flexion) of a load (Mitchell and Sale, 2011). If five repetitions were completed, the weight of the load was increased by $14-18 \mathrm{~kg}$. The participant's $5 \mathrm{RM}$ was determined within five trials. There was a five minute rest interval between the trials.

Each participant's individual drop jump height was determined according to Byrne et al. (2010). First, the participant completed three VJs with a $20 \mathrm{~s}$ rest between VJs keeping their hands on the waist during VJ. The three VJ heights (VJH) were averaged to calculate 50, 75, 100, 125 and $150 \%$ of the VJH for the drop jump height (McCaulley et al., 2007). Each participant was randomly assigned to perform three drop jump trials from each of the five drop heights with a 20 $\mathrm{s}$ rest between trials and a 2 min rest between changed drop heights. The participants were instructed to keep their hands on the waist and to step off the platform with the leading parallel leg to avoid any initial upward propulsion and ensure the starting drop heights. They were also instructed to jump as quickly and high as possible upon landing, keeping the knees and ankles fully extended upon leaving the ground and assuming a similar extended position when landing. All of the participants were required to land with one foot on each of the two force plates for the completion of the drop jump. After testing, the ratio of the drop jump height and drop jump ground contact time were calculated and averaged for the three trials of each drop height. The greatest ratio of drop jump height and drop jump ground contact time found at a drop height was assigned as the individual PE drop jump 
height.

After the 5 RM squat and drop jump testing, five different PAP protocols were performed on five separate days. A standardised warm up, comprised of light intensity cycling for five minutes at slow pace and a series of dynamic stretches for five minutes, was performed before testing. After the warm-up, each participant performed three VJs with a $20 \mathrm{~s}$ rest between each trial as a baseline (pre-test) (Jensen and Ebben, 2003). After the three VJ pre-tests, a one minute rest was taken, followed by one of five different PAP protocols (three single conditioning exercises and two complex training modalities): RE, PE, WBV, CT and VCT. RE consisted of one set of five repetitions of $5 \mathrm{RM}$ squat $\left(90^{\circ}\right.$ of knee flexion) without rest between repetitions. PE was comprised of one set of five repetitions of each individual's drop jump height with a $5 \mathrm{~s}$ rest between repetitions. WBV consisted of three exposures $\times 10 \mathrm{~s}$ with $1 \mathrm{~min}$ of rest between exposures, a $30 \mathrm{~Hz}$ frequency and $2.5 \mathrm{~mm}$ amplitude (peak to peak). The participants placed their hands on the hips and squatted $\left(90^{\circ}\right.$ of knee flexion) on a vibration plate, which administered a pivotal vibration (TVR-4900, Tonic Fitness Technology, INC., Taipei, Taiwan). CT consisted of RE followed by PE with a 4 min rest. VCT included WBV followed by PE with a 4 min rest period. Lastly, the participants had a 4 min rest after completion of the PAP protocol and then performed three VJs (post-test). A schematic diagram of the experimental procedure is shown in Figure 1.

\section{Instrumentation}

Two force plates (BP600900, AMTI Inc., Watertown, MA, USA) were used to record VJs at a $2000 \mathrm{~Hz}$ sampling rate. All data were transformed and analysed using MotionMonitor software (Innovative Sports Training, Inc., Chicago. IL. USA).

\section{Data analysis}

The drop jump height and $\mathrm{VJH}$ were calculated with the formula: $1 / 8 \mathrm{gT}^{2}\left(\mathrm{~g}=9.81 \mathrm{~m} \cdot \mathrm{s}^{-2}\right.$; $\mathrm{T}=$ flight time of drop jump height or $\mathrm{VJH})$. The dependent variable was averaged from the three VJs for further analyses.

\section{Statistical Analyses}

Statistical analyses were performed using SPSS software version 12 (SPSS Inc., Chicago, IL, USA). Descriptive statistics (Mean \pm SD) was used to determine the participant and dependent variable characteristics. A repeated-measures twoway (five PAP protocols $\times$ two testing times) ANOVA was performed for the dependent variable. The level of significance was set at $p<$ 0.05 . Whenever a significant main effect occurred, Fisher's least significant difference was used for post hoc tests. Partial Eta Squared $\left(\eta p^{2}\right)$ values were calculated to estimate effect size which was considered either small $(0.01)$, moderate $(0.06)$ or large (0.15) (Cohen, 2013; Naclerio et al., 2014). Intraclass correlation coefficients (ICC) were high for all jump pre-tests (ICC $=0.866$; $95 \%$ confidence intervals $=0.712-0.959)$.

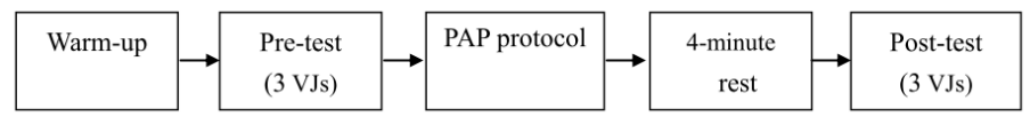

Figure 1

Schematic diagram of the experimental procedures.

Three VJs were performed before (pre-test)

and after (post-test) the PAP protocol, which included PE, WBV, RE, CT

and VCT. Each PAP protocol was performed on separate days. 


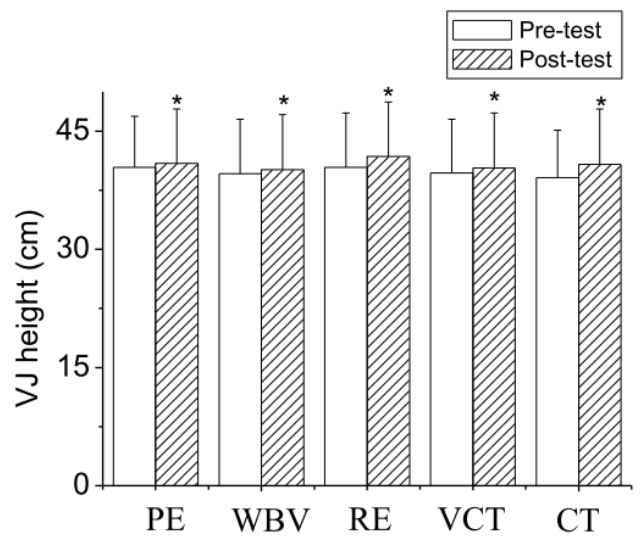

Figure 2

VJ height before and after the five PAP protocols.

* Significantly different from the pre-test. Abbreviations:

$P E=$ plyometric exercise; $W B V=$ whole body vibration;

$R E=$ resistance exercise;

$V C T=$ vibration complex training; $C T=$ complex training.

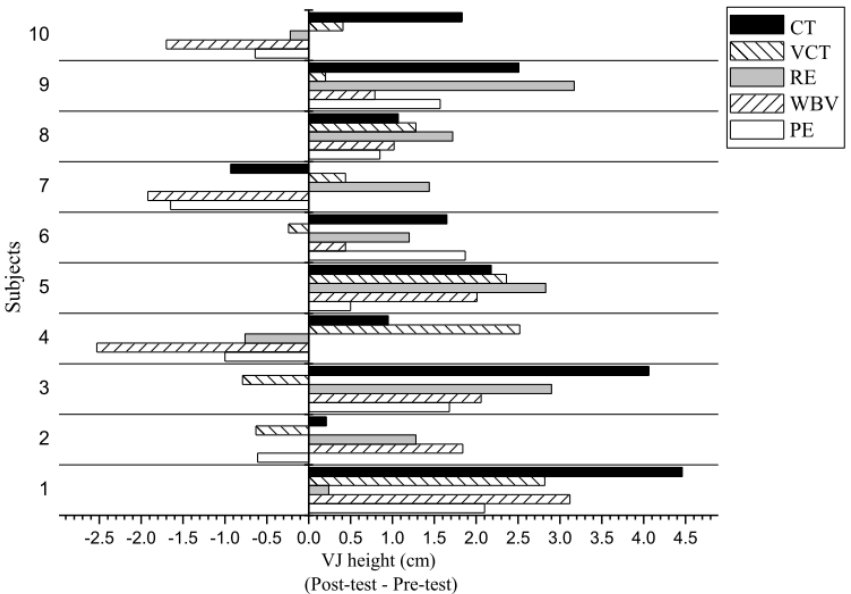

Figure 3

Individual VJ height after five PAP protocols, compared with the pre-test. Abbreviations: $P E=$ plyometric exercise; $W B V=$ whole body vibration; $R E=$ resistance exercise; $V C T=$ vibration complex training; $C T=$ complex training. 


\section{Results}

No significant interaction was found between the five PAP protocols and two testing times for the dependent variable. Significant main effects between the two testing times were found in $\mathrm{VJH}$ $(p=0.015)$ and with a large effect size $\left(\eta p^{2}=0.502\right)$. The post hoc analysis indicated that the post-test values were significantly greater compared to the pre-test (Figure 2). Individual changes of each participant were observed in the VJ performance after five PAP protocols (Figure 3). Large individual responses were also found for VJ performance. Some participants responded positively to all PAP protocols (participant 1, 5, 8, 9), whereas other participants responded only to selected PAP protocols.

\section{Discussion}

The aims of the present study were (a) to determine whether CT and VCT would improve the individual phenomenon of PAP for every athlete in a team setting; and (b) to compare the acute effect of single conditioning exercise (RE, PE and $\mathrm{WBV}), \mathrm{CT}$ and VCT on VJ performance. It was hypothesized CT and VCT would lead to a better VJ performance than a single conditioning exercise and improve the individual phenomenon of PAP. This study had two main findings. Firstly, $\mathrm{CT}$ and VCT failed to improve the individual phenomenon of PAP for every athlete in a team setting. Secondly, CT and VCT failed to induce a greater VJ performance when compared to single conditioning exercises.

Previously, only two studies had compared the effect of CT, PE and RE on PAP prior to judo and taekwondo performance. Miarka et al. (2011) found a significant improvement in specific judo performance after PE (ten sets of three consecutive jumps), however, RE (five sets of one squat repetition at 95\% $1 \mathrm{RM}$ ) and CT (combining three sets of two repetitions of the participant's $90 \% 1 \mathrm{RM}$ squat with five consecutive jumps) groups showed no significant improvement. da Silva Santos et al. (2015) found a significant improvement in taekwondo performance after CT (combining three sets of two repetitions of $95 \%$ 1RM half-squat with four vertical jumps), however, RE (three sets of one repetition 95\% 1RM half-squat) and PE (three sets of ten repetitions of the vertical jump) groups showed no significant improvement. It is difficult to compare the research of Miarka et al. (2011) and da Silva Santos et al. (2015) with the present study because of different protocols used (i.e., intensity, volume, recovery time and the type of conditioning exercise) and different types of subsequent activities used to examine the PAP benefit (Tillin and Bishop, 2009). The lack of improvement in VJ performance during $\mathrm{CT}$ and VCT compared to single conditioning exercises may be explained by the individual phenomenon of PAP (Comyns et al., 2006), for example, participant 9 showed the greatest improvement in RE, participant 2 improved the most in WBV and for participant 10 the greatest improvement was observed in CT.

Although this study found that CT and VCT as well as single conditioning exercises produced similar group PAP benefits, it cannot be stated that $\mathrm{CT}$ and VCT along with single conditioning exercises were effective for every athlete observed. For example, performance of participant 2 was decreased in PE and VCT, performance of participant 3 worsened in VCT, while participant 4 showed a decreased performance in PE, WBV and RE. Previous research has indicated that the rest interval between the conditioning exercise and subsequent activity should be determined individually (Comyns et al., 2006). However, the current study used a blanket four minutes for the time between exercises in the complex conditions and time between the conditioning exercise and subsequent activity. The decrease that some individuals showed in VJ performance might be due to inappropriate duration of the rest period that would have allowed to take advantage of PAP (intensity $\times$ volume); perhaps a decrease or an increase in the rest interval would be required to improve performance (e.g. three minutes or five minutes).

The participant characteristics that have been reported to affect an individual's PAP response include muscular strength, fibre-type distribution, training level, gender and a powerstrength ratio, along with variables that have not been identified yet (e.g. body composition) (Robbins, 2005). Several investigations have reported that stronger individuals were able to elicit greater PAP than their weaker counterparts (Chiu et al., 2003; Duthie et al., 2002). This study found that $\mathrm{CT}$ and VCT failed to improve the 
individual phenomenon of PAP. It is possible that strength levels in some participants might be low and thus, they could not effectively utilize PAP to improve VJ performance. In addition, other variables that have not yet been identified may have caused some additional discrepancies between individuals.

Because previous investigations have reported that single conditioning exercises show different individual PAP responses due to conditioning levels, researchers have suggested that coaches evaluate the athletes before assigning a single conditioning exercise as part of their warm-up routine (Batista et al., 2011; Lim et al., 2013; Bogdanis et al., 2014; Weber et al., 2008). In addition, the current study further demonstrated that CT and VCT also produced different individual PAP responses. Therefore, the practical applicability of PAP with respect to increasing athletic performances within a team setting is quite controversial. This dilemma may be resolved by decreasing fatigue, although future studies should examine the effect of low-intensity and low-volume activity with appropriate recovery time on the individual phenomenon of PAP.

A limitation of our study is that the intensity and volume of CT and VCT were higher than in the separate single conditioning exercises. Different intensity and volume within CT, VCT and single conditioning exercises may have confounded our results which showed similar PAP effects in all conditioning exercises.

\section{Conclusions}

This study showed that most athletes improved their vertical jump performance after complex training, vibration complex training and single conditioning exercises. However, it is not recommended for coaches to arrange the exercises in a team setting because some athletes decreased their performance, while others showed improvement.

\section{Acknowledgements}

This study was supported by the National Science Council of Taiwan (NSC 101-2410-H-034-054).

\section{References}

Arabatzi F, Kellis E, Saez-Saez De Villarreal E. Vertical jump biomechanics after plyometric, weight lifting, and combined (weight lifting + plyometric) training. J Strength Cond Res, 2010; 24: 2440-2448

Barroso R, Silva-Batista C, Tricoli V, Roschel H, Ugrinowitsch, C. The effects of different intensities and durations of the general warm-up on leg press 1RM. J Strength Cond Res, 2013; 27: 1009-1013

Bogdanis GC, Tsoukos A, Veligekas P, Tsolakis C, Terzis G. Effects of muscle action type with equal impulse of conditioning activity on postactivation potentiation. J Strength Cond Res, 2014; 28: 2521-2528

Byrne PJ, Moran K, Rankin P, Kinsella S. A comparison of methods used to identify 'optimal' drop height for early phase adaptations in depth jump training. J Strength Cond Res, 2010; 24: 2050-2055.

Baechle TR, Earle RW. Essentials of Strength Training and Conditioning (5th ed.). Champaign: Human Kinetics, 394-396; 2008

Comyns TM, Harrison AJ, Hennessy LK, Jensen RL. The optimal complex training rest interval for athletes from anaerobic sports. J Strength Cond Res, 2006; 20: 471-476

Chattong C, Brown LE, Coburn JW, Noffal GJ. Effect of a dynamic loaded warm-up on vertical jump performance. J Strength Cond Res, 2010; 24: 1751-1754

Chen ZR, Wang YH, Peng HT, Yu CF, Wang MH. The acute effect of drop jump protocols with different volumes and recovery time on countermovement jump performance. J Strength Cond Res, 2013; 27: $154-158$

Cochrane DJ, Stannard SR, Sargeant AJ, Rittweger J. The rate of muscle temperature increase during acute whole-body vibration exercise. Eur J Appl Physiol, 2008; 103: 441-448

Cohen J. Statistical power analysis for the behavioral sciences. Hillsdale: Routledge; 2013 
Cormie P, Deane RS, Triplett NT, McBride JM. Acute effects of whole-body vibration on muscle activity, strength, and power. J Strength Cond Res, 2006; 20: 257-261

Chiu LZ, Fry AC, Weiss LW, Schilling BK, Brown LE, Smith SL. Postactivation potentiation response in athletic and recreationally trained individuals. J Strength Cond Res, 2003; 17: 671-677

da Silva Santos JF, Valenzuela TH, Franchini E. Can different conditioning activities and rest intervals affect the acute performance of taekwondo turning kick? J Strength Cond Res, 2015; 29: 1640-1647

Dallas G, Smirniotou A, Tsiganos G, Tsopani D, Di Cagno A, Tsolakis C. Acute effect of different stretching methods on flexibility and jumping performance in competitive artistic gymnasts. J Sports Med Phys Fitness, 2014; 54: 683-690

Duthie GM, Young WB, Aitken DA. The acute effects of heavy loads on jump squat performance: an evaluation of the complex and contrast methods of power development. J Strength Cond Res, 2002; 16: $530-538$

Fatouros IG, Jamurtas AZ, Leontsini D, Taxildaris K, Aggelousis N, Kostopoulos N, Buckenmeyer P. Evaluation of plyometric exercise training, weight training, and their combination on vertical jumping performance and leg strength. J Strength Cond Res, 2000; 14: 470-476

Gołaś A, Maszczyk A, Zajac A, Mikołajec K, Stastny P. Optimizing Post Activation Potentiation for Explosive Activities in Competitive Sports. J Hum Kinet, 2016; 42: 95-106

Hanson ED, Leigh S, Mynark RG. Acute effects of heavy- and light-load squat exercise on the kinetic measures of vertical jumping. J Strength Cond Res, 2007; 21: 1012-1017

Jensen RL, Ebben WP. Kinetic analysis of complex training rest interval effect on vertical jump performance. J Strength Cond Res, 2003; 17: 345-349

Kilduff LP, Bevan HR, Kingsley MI, Owen NJ, Bennett MA, Bunce PJ, Hore AM, Maw JR, Cunningham DJ. Postactivation potentiation in professional rugby players: optimal recovery. J Strength Cond Res, 2007; 21: $1134-1138$

Kotzamanidis C, Chatzopoulos D, Michailidis C, Papaiakovou G, Patikas D. The effect of a combined highintensity strength and speed training program on the running and jumping ability of soccer players. $J$ Strength Cond Res, 2005; 19: 369-375

Lamont HS, Cramer JT, Bemben DA, Shehab RL, Anderson MA, Bemben MG. The acute effect of wholebody low-frequency vibration on countermovement vertical jump performance in college-aged men. $J$ Strength Cond Res, 2010; 24: 3433-3442

Lim JJ, Kong PW. Effects of isometric and dynamic postactivation potentiation protocols on maximal sprint performance. J Strength Cond Res, 2013; 27: 2730-2736

McCann MR, Flanagan SP. The effects of exercise selection and rest interval on postactivation potentiation of vertical jump performance. J Strength Cond Res, 2010; 24: 1285-1291

McCaulley GO, Cormie P, Cavill MJ, Nuzzo JL, Urbiztondo ZG, McBride JM. Mechanical efficiency during repetitive vertical jumping. Eur J Appl Physiol, 2007; 101: 115-123

McLellan CP, Lovell DI, Gass GC. The role of rate of force development on vertical jump performance. J Strength Cond Res, 2011; 25: 379-385

Miarka B, Del Vecchio FB, Franchini E. Acute effects and postactivation potentiation in the Special Judo Fitness Test. J Strength Cond Res, 2011; 25: 427-431

Mitchell CJ, Sale DG. Enhancement of jump performance after a 5-RM squat is associated with postactivation potentiation. Eur J Appl Physiol, 2011; 111: 1957-1963

Naclerio F, Faigenbaum AD, Larumbe-Zabala E, Ratamess NA, Kang J, Friedman P, Ross RE. Effectiveness of different postactivation potentiation protocols with and without whole body vibration on jumping performance in college athletes. J Strength Cond Res, 2014; 28: 232-239 
Robbins DW. Postactivation potentiation and its practical applicability. J Strength Cond Res, 2005; 19: 453-458.

SaezSaez de Villarreal E, Gonzalez-Badillo JJ, IzquierdoM. Optimal warm-up stimuli of muscle activation to enhance short and long-term acute jumping performance. Eur J Appl Physiol, 2007; 100: 393-401

Till KA, Cooke C. The effects of postactivation potentiation on sprint and jump performance of male academy soccer players. J Strength Cond Res, 2009; 23: 1960-1967

Tillin NA, Bishop D. Factors modulating post-activation potentiation and its effect on performance of subsequent explosive activities. Sports Med, 2009; 39: 147-166

Wang HH, Chen WH, Liu C, Yang WW, Huang MY, Shiang TY. Whole-body vibration combined with extraload training for enhancing the strength and speed of track and field athletes. J Strength Cond Res, 2014; 28: $2470-2477$

Weber KR, Brown LE, Coburn JW, Zinder SM. Acute effects of heavy-load squats on consecutive squat jump performance. J Strength Cond Res, 2008; 22: 726-730

\section{Corresponding author:}

\section{Hsien-Te Peng}

Address: 55, Hwa-Kang Road, Yang-Ming-Shan, Taipei, Taiwan 11114, R. O. C.

Affiliation: Department of Physical Education Chinese Culture University, Taipei, Taiwan

Tel: 886-2-28610511-43260 or 43292

Cell: 886-930379219

E-mail address: pxd@ulive.pccu.edu.tw 